\title{
Disturbance Decoupling with Preview for Two-Dimensional Systems *
}

\author{
L. Ntogramatzidis ${ }^{*}$ M. Cantoni ${ }^{* *}$ R. Yang ${ }^{* * *}$ \\ * Department of Electrical and Electronic Engineering, \\ The University of Melbourne, Parkville, VIC 3010, Australia \\ (e-mail: lnt@ee.unimelb.edu.au) \\ ** Department of Electrical and Electronic Engineering, \\ The University of Melbourne, Parkville, VIC 3010, Australia \\ (e-mail:m.cantoni@ee.unimelb.edu.au) \\ *** School of Information Science and Technology, \\ Sun Yat-Sen University, Guangzhou 510275, P.R. China \\ (e-mail: yangran@mail.sysu.edu.cn)
}

\begin{abstract}
In this paper a solution is given to the exact disturbance decoupling problem for twodimensional (2-D) systems, whereby the control action consists of a static local state feedback and a preview function of the signal to be rejected. Importantly, stability of the closed loop is taken into account.
\end{abstract}

\section{INTRODUCTION}

The notion of controlled invariance introduced by Basile and Marro in [2] is the cornerstone of the so-called geometric approach to control theory for LTI systems. The most celebrated control application of this concept is the disturbance decoupling problem, solved for the first time in [2]. Disturbance decoupling with the extra requirement of closed-loop stability was addressed for the first time by Wonham and Morse in [19] via the introduction of $(A, B)$ stabilizability subspaces. An improved solution to the same problem was suggested by Basile and Marro in [3], relying on the concept of self-bounded controlled invariance to avoid eigenspace computation. This solution was later shown to be the best one in terms of pole placement, [12]. Many important variations of the classic decoupling problem were proposed in the literature in the last thirty years. The one that is most relevant for this paper is the so-called disturbance decoupling with PID control law, $[18,4,1]$. In the discrete-time case, this problem is also referred to as disturbance decoupling with preview, since the control law is allowed to include - in addition to the standard proportional state feedback component - feedforward terms depending on 'future' values of the disturbance up to the present.

In the last two decades, many valuable results have been achieved in the attempt to develop a geometric theory for 2-D systems, $[5,9,10,13,14,15]$. In particular, a geometric approach for 2-D systems was first introduced in [5] to treat 2-D decoupling problems of nonmeasurable and measurable disturbances, but without a guarantee of stability. In [15], new geometric techniques for internal and external stabilisation of controlled invariant and outputnulling subspaces were developed. This lead to a new solution for the two aforementioned decoupling problems, while achieving asymptotic stability of the closed-loop.

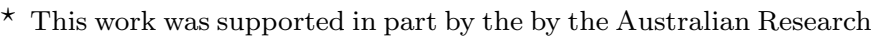
Council (DP0664789).
In this paper the disturbance decoupling with preview is extended for the first time to 2-D causal systems. Its solution is carried out by recasting this problem into a full information problem. This contrivance enables the structural solvability condition to be easily stated in terms of the matrices of a suitably defined extended system. However, the stability condition must be addressed independently, and here it is captured in terms of the stability property of an output-nulling subspace of the original system.

Notation. The symbol $\mathbf{0}_{n}$ stands for the origin of the vector space $\mathbb{R}^{n}$. The image and the kernel of the linear map associated with multiplication by a matrix $M \in$ $\mathbb{R}^{n \times m}$ are denoted by $\operatorname{im} M \subseteq \mathbb{R}^{n}$ and $\operatorname{ker} M \subseteq \mathbb{R}^{m}$, respectively. The $n \times m$ zero matrix is denoted by $0_{n \times m}$ and the $n \times n$ identity matrix is denoted by $I_{n}$. Given a matrix $M$, the symbols $M^{\top}$ and $M^{\dagger}$ denote the transpose and the Moore-Penrose pseudoinverse of $M$, respectively. Given the subspace $\mathcal{S}$, the symbol $\mathcal{S}^{2}$ stands for the Cartesian product $\mathcal{S} \times \mathcal{S}$,

\section{PROBLEM STATEMENT}

Consider a Fornasini-Marchesini (FM) model

$$
\begin{aligned}
x_{i+1, j+1}= & A_{1} x_{i+1, j}+A_{2} x_{i, j+1}+B_{1} u_{i+1, j}+B_{2} u_{i, j+1} \\
& +H_{1} w_{i+1, j}+H_{2} w_{i, j+1}, \\
y_{i, j}= & C x_{i, j}+D u_{i, j}+G w_{i, j},
\end{aligned}
$$

where for all $i, j \in \mathbb{Z}, x_{i, j} \in \mathbb{R}^{n}$ is the local state, $u_{i, j} \in \mathbb{R}^{m}$ is the control input, $w_{i, j} \in \mathbb{R}^{d}$ is a disturbance to be decoupled from the output $y_{i, j} \in \mathbb{R}^{p}$. The matrices appearing in (1) have sizes compatible with these signals. We identify the system $\left(A_{1}, A_{2},\left[B_{1} H_{1}\right],\left[B_{2} H_{2}\right], C,[D G]\right)$ with the symbol $\Sigma$.

For $k \in \mathbb{Z}$, we define the separation $\operatorname{sets}^{1} \mathbb{S}_{k} \triangleq\{(i, j) \in$

\footnotetext{
1 As shown in [7], other separation sets can be defined so that boundary conditions specified over them uniquely determine a local-
} 
$\mathbb{Z} \times \mathbb{Z} \mid i+j=k\}$, along with the so-called global state on $\mathbb{S}_{k}$ as $\mathcal{X}_{k} \triangleq\left\{x_{i, j} \mid(i, j) \in \mathbb{S}_{k}\right\}$, see [6]. Similarly, we can define the global control $\mathcal{U}_{k} \triangleq\left\{u_{i, j} \mid(i, j) \in \mathbb{S}_{k}\right\}$, the global disturbance $\mathcal{W}_{k} \triangleq\left\{w_{i, j} \mid(i, j) \in \mathbb{S}_{k}\right\}$ and the global output $\mathcal{Y}_{k} \triangleq\left\{y_{i, j} \mid(i, j) \in \mathbb{S}_{k}\right\}$ on the separation sets. As such, the boundary conditions usually associated with (1) take the form $x_{i, j}=b_{i, j}$ for $(i, j) \in \mathbb{S}_{0}$ for some constants $b_{i, j} \in \mathbb{R}^{n}$ for $(i, j) \in \mathbb{S}_{0}$. This uniquely defines $\mathcal{X}_{k}$ for all $k>0$ given $\mathcal{U}_{h}$ and $\mathcal{W}_{h}$ for all $0 \leq h<k$.

Given a subspace $\mathcal{S}$, by a $\mathcal{S}$-valued boundary condition we intend $x_{i, j} \in \mathcal{S}$ for all $(i, j) \in \mathbb{S}_{0}$. By defining $\left\|\mathcal{X}_{r}\right\| \triangleq \sup _{n \in \mathbb{Z}}\left\|x_{r-n, n}\right\|$, we recall that system (1) and therefore, with a slight abuse of nomenclature, the pair $\left(A_{1}, A_{2}\right)$ - is asymptotically stable if, for finite $\left\|\mathcal{X}_{0}\right\|$ and with both inputs set to zero, the sequence $\left\{\left\|\mathcal{X}_{i}\right\|\right\}_{i=0}^{\infty}$ converges to zero.

It is well-known that the pair $\left(A_{1}, A_{2}\right)$ is asymptotically stable if, and only if,

$$
\operatorname{det}\left(I_{n}-A_{1} z_{2}-A_{2} z_{1}\right) \neq 0 \quad \forall\left(z_{1}, z_{2}\right) \in \mathfrak{P}
$$

where $\mathfrak{P}=\left\{\left(\zeta_{1}, \zeta_{2}\right) \in \mathbb{C} \times \mathbb{C}|| \zeta_{1} \mid \leq 1\right.$ and $\left.\left|\zeta_{2}\right| \leq 1\right\}$ is the unit bidisc [6]. A simple sufficient condition that can be used to check asymptotic stability of the pair $\left(A_{1}, A_{2}\right)$ is the one proposed in [8]: The pair $\left(A_{1}, A_{2}\right)$ is asymptotically stable if two symmetric positive definite matrices $P_{1}$ and $P_{2}$ exist such that:

$$
\left[\begin{array}{cc}
P_{1} & 0 \\
0 & P_{2}
\end{array}\right]-\left[\begin{array}{c}
A_{1}^{\top} \\
A_{2}^{\top}
\end{array}\right]\left(P_{1}+P_{2}\right)\left[\begin{array}{ll}
A_{1} & A_{2}
\end{array}\right]>0 .
$$

Problem 2.1. (Disturbance decoupling with preview) Given $N, M \in \mathbb{N}$, find matrices $F \in \mathbb{R}^{m \times n}$ and $S_{k, l} \in$ $\mathbb{R}^{m \times d}$, for $(k, l) \in[0, N] \times[0, M]$, so that the system

$$
\begin{gathered}
x_{i+1, j+1}=\left(A_{1}+B_{1} F\right) x_{i+1, j}+\left(A_{2}+B_{2} F\right) x_{i, j+1} \\
+B_{1} \varphi_{i+1, j}+B_{2} \varphi_{i, j+1}+H_{1} w_{i+1, j}+H_{2} w_{i, j+1},(4) \\
y_{i, j}=(C+D F) x_{i, j}+D \varphi_{i, j}+G w_{i, j}
\end{gathered}
$$

obtained by imposing the control action

$$
u_{i, j}=F x_{i, j}+\varphi_{i, j}
$$

with $\varphi_{i, j} \triangleq \sum_{k=0}^{N} \sum_{l=0}^{M} S_{k, l} w_{i+k, j+l}$, on the system dynamics (1), yields a global output sequence $\left\{\mathcal{Y}_{i}\right\}_{i=0}^{\infty}$ with elements that converge to zero for any global-state boundary condition $\mathcal{X}_{0}$ and any global disturbance $\left\{\mathcal{W}_{i}\right\}_{i=0}^{\infty}$.

By linearity, Problem 2.1 is equivalent to requiring that

- with the boundary conditions set to zero (i.e., $x_{i, j}=0$ for $\left.(i, j) \in \mathbb{S}_{0}\right)$, the output generated by (4) satisfy $y_{i, j}=0$ for all $i+j \geq 0$ and any global disturbance $\left\{\mathcal{W}_{i}\right\}_{i=0}^{\infty}$

- the pair $\left(A_{1}+B_{1} F, A_{2}+B_{2} F\right)$ be asymptotically stable, to ensure dissipation of the effect of non-zero boundary conditions on the output.

state trajectory solution of (6) over a region of $\mathbb{Z} \times \mathbb{Z}$. A useful example is the separation set $\mathbb{S}_{k} \triangleq\{(i, j) \in\{0\} \times[1, \infty) \cup[1, \infty) \times\{0\}\}$, which with corresponding boundary conditions uniquely determines $x_{i, j}$ for $(i, j) \in \mathbb{N} \times \mathbb{N}$.

\section{GEOMETRIC BACKGROUND FOR 2-D SYSTEMS}

Before presenting the solution of Problem 2.1, we need some geometric preliminaries for $2-\mathrm{D}$ systems. These are mainly taken from [15]. We begin by considering the autonomous FM system

$$
x_{i+1, j+1}=A_{1} x_{i+1, j}+A_{2} x_{i, j+1},
$$

The subspace $\mathcal{J}$ of $\mathbb{R}^{n}$ is said to be $\left(A_{1}, A_{2}\right)$-invariant if it is simultaneously $A_{1}$ and $A_{2}$-invariant, i.e., if $A_{1} \mathcal{J} \subseteq \mathcal{J}$ and $A_{2} \mathcal{J} \subseteq \mathcal{J}$. If $\mathcal{J}$ is a $q$-dimensional $\left(A_{1}, A_{2}\right)$-invariant, by choosing a nonsingular matrix $T=\left[T_{1} T_{2}\right] \in \mathbb{R}^{n \times n}$ where the columns of $T_{1}$ span $\mathcal{J}$, we find that

$$
T^{-1} A_{i} T=\left[\begin{array}{cc}
A_{i}^{(1,1)} & A_{i}^{(1,2)} \\
0_{(n-q) \times q} & A_{i}^{(2,2)}
\end{array}\right] \quad \text { for } \quad i=1,2 .
$$

Thus, (6) can be written in the new coordinates described as $\left[\begin{array}{c}x_{i, j}^{\prime} \\ x_{i, j}^{\prime \prime}\end{array}\right]=T^{-1} x_{i, j}$, so that

$$
\begin{gathered}
x_{i+1, j+1}^{\prime}=A_{1}^{(1,1)} x_{i+1, j}^{\prime}+A_{1}^{(1,2)} x_{i+1, j}^{\prime \prime} \\
+A_{2}^{(1,1)} x_{i, j+1}^{\prime}+A_{2}^{(1,2)} x_{i, j+1}^{\prime \prime}, \\
x_{i+1, j+1}^{\prime \prime}=A_{1}^{(2,2)} x_{i+1, j}^{\prime \prime}+A_{2}^{(2,2)} x_{i, j+1}^{\prime \prime} .
\end{gathered}
$$

As in the $1-\mathrm{D}$ case, given an $\left(A_{1}, A_{2}\right)$-invariant subspace $\mathcal{J}$ for (6), any $\mathcal{J}$-valued boundary condition gives rise to a local state trajectory such that $x_{i, j} \in \mathcal{J}$ for all $i, j$ such that $i+j \geq 0$. By (8-9) and (2), it is easy to see that asymptotic stability of (6) can be "split" into two parts with respect to the invariant subspace $\mathcal{J}$. Indeed, (8-9) is asymptotically stable if and only if $\left(A_{1}^{(1,1)}, A_{2}^{(1,1)}\right)$ and $\left(A_{1}^{(2,2)}, A_{2}^{(2,2)}\right)$ are such. The $\left(A_{1}, A_{2}\right)$-invariant subspace $\mathcal{J}$ is said to be:

- inner stable if $\left(A_{1}^{(1,1)}, A_{2}^{(1,1)}\right)$ is asymptotically stable;

- outer stable if $\left(A_{1}^{(2,2)}, A_{2}^{(2,2)}\right)$ is asymptotically stable.

It turns out that the pair $\left(A_{1}, A_{2}\right)$ is asymptotically stable if and only if any $\left(A_{1}, A_{2}\right)$-invariant subspace is inner and outer stable.

Now, we turn our attention to the nonautonomous Fornasini-Marchesini system

$$
\begin{aligned}
x_{i+1, j+1} & =A_{1} x_{i+1, j}+A_{2} x_{i, j+1}+B_{1} u_{i+1, j}+B_{2} u_{i, j+1}(10) \\
y_{i, j} & =C x_{i, j}+D u_{i, j}
\end{aligned}
$$

The boundary conditions associated with (10-11) can still be assigned by specifying the global state over $\mathbb{S}_{0}$. The subspace $\mathcal{V} \subseteq \mathbb{R}^{n}$ is output-nulling for (10-11) if

$$
\left[\begin{array}{c}
A_{1} \\
A_{2} \\
C
\end{array}\right] \mathcal{V} \subseteq\left(\mathcal{V}^{2} \times \mathbf{0}_{p}\right)+\operatorname{im}\left[\begin{array}{c}
B_{1} \\
B_{2} \\
D
\end{array}\right]
$$

[5]. Let $\mathcal{V}$ be a subspace of $\mathbb{R}^{n}$ and let $V$ be a basis matrix of $\mathcal{V}$. The following are equivalent [15]:

- The subspace $\mathcal{V}$ is output-nulling for (10-11);

- There exist $X$ and $\Omega$ such that

$$
\left[\begin{array}{c}
A_{1} \\
A_{2} \\
C
\end{array}\right] V=\left[\begin{array}{ll}
V & 0 \\
0 & V \\
0 & 0
\end{array}\right] X+\left[\begin{array}{c}
B_{1} \\
B_{2} \\
D
\end{array}\right] \Omega
$$


- There exist $F$ such that $\mathcal{V}$ is $\left(A_{1}+B_{1} F, A_{2}+B_{2} F\right)$ invariant and $\mathcal{V} \subseteq \operatorname{ker}(C+D F)$;

- There exist $F$ and $X$ such that

$$
\left[\begin{array}{c}
A_{1}+B_{1} F \\
A_{2}+B_{2} F \\
C+D F
\end{array}\right] V=\left[\begin{array}{ll}
V & 0 \\
0 & V \\
0 & 0
\end{array}\right] X,
$$

The set of matrices $X$ and $\Omega$ satisfying the linear equation (13) can be parameterised by

$$
\left[\begin{array}{l}
X \\
\Omega
\end{array}\right]=W^{\dagger}\left[\begin{array}{c}
A_{1} \\
A_{2} \\
C
\end{array}\right] V+H K
$$

where $W \triangleq\left[\begin{array}{ccc}V & 0 & B_{1} \\ 0 & V & B_{2} \\ 0 & 0 & D\end{array}\right], H$ is a basis matrix of $\operatorname{ker} W$ and $K$ is an arbitrary matrix of suitable size. Any matrix $F$ such that (14) holds for some $X$ is called a friend of $\mathcal{V}$. Given a $\mathcal{V}$-valued boundary condition for (10-11), a control action $u_{i, j}=F x_{i, j}$, where $F$ satisfies (14), is such that $x_{i, j} \in \mathcal{V}$ and $y_{i, j}=0$ for all $i, j$ such that $i+j \geq 0$. To see this, it suffices to substitute $u_{i, j}=F x_{i, j}$ in (10-11) to get

$$
\begin{aligned}
x_{i+1, j+1} & =\left(A_{1}+B_{1} F\right) x_{i+1, j}+\left(A_{2}+B_{2} F\right) x_{i, j+1} \\
y_{i, j} & =(C+D F) x_{i, j} .
\end{aligned}
$$

When $x_{i+1, j}$ and $x_{i, j+1}$ belong to $\mathcal{V}$, so does $x_{i+1, j+1}$ in view of (14). As a result, for any $\mathcal{V}$-valued boundary condition it is found that $x_{i, j} \in \mathcal{V}$ and $y_{i, j}=0$ for all $i, j$ such that $i+j \geq 0$, since $\mathcal{V} \subseteq \operatorname{ker}(C+D F)$. Hence, the control function maintaining the output at zero and the local state on $\mathcal{V}$ can always be expressed in feeback form as in the 1-D setting. The output-nulling subspace $\mathcal{V}$ is said to be inner stabilisable (resp. outer stabilisable) if there exists a friend $F$ such that $\mathcal{V}$ is an inner stable (resp. outer stable) $\left(A_{1}+B_{1} F, A_{2}+B_{2} F\right)$-invariant. It is easily seen that the set of friends of $\mathcal{V}$ are parameterised as the solutions of the linear equation $\Omega=-F V$, where $\Omega$ satisfies (13) for some matrix $X$. In particular, the solutions of $\Omega=-F V$ can be written as

$$
F=F_{\Omega}+\Lambda, \quad F_{\Omega} \triangleq-\Omega\left(V^{\top} V\right)^{-1} V^{\top}
$$

where $\Omega$ satisfies (13) for some matrix $X$ and $\Lambda$ is any matrix of suitable size such that $\Lambda V=0$, see [15]. Writing the local state equation of the autonomous system obtained by applying $u_{i, j}=F x_{i, j}$, with $F=F_{\Omega}+$ $\Lambda$, to (10) in a new set of coordinates characterised by $T=\left[\begin{array}{ll}T_{1} & T_{2}\end{array}\right]$ with $\operatorname{im} T_{1}=\mathcal{V}$, yields

$$
\begin{gathered}
{\left[\begin{array}{c}
x_{i+1, j+1}^{\prime} \\
x_{i+1, j+1}^{\prime \prime}
\end{array}\right]=\left[\begin{array}{cc}
M_{1}^{(1,1)} & M_{1}^{(1,2)} \\
0 & M_{1}^{(2,2)}
\end{array}\right]\left[\begin{array}{l}
x_{i+1, j}^{\prime} \\
x_{i+1, j}^{\prime \prime}
\end{array}\right]} \\
+\left[\begin{array}{cc}
M_{2}^{(1,1)} & M_{2}^{(1,2)} \\
0 & M_{2}^{(2,2)}
\end{array}\right]\left[\begin{array}{l}
x_{i, j+1}^{\prime} \\
x_{i, j+1}^{\prime \prime}
\end{array}\right],
\end{gathered}
$$

where $M_{i} \triangleq A_{i}+B_{i} F$. Different values of $\Omega$ and $\Lambda$ in (17) give rise to different values for the matrices $M_{k}^{(i, j)}$. However, it is shown in [15] that the pair $\left(M_{1}^{(1,1)}, M_{2}^{(1,1)}\right)$ only depends on $F_{\Omega}$ while the pair $\left(M_{1}^{(2,2)}, M_{2}^{(2,2)}\right)$ only depends on $\Lambda$. Therefore, we can independently choose $F_{\Omega}$ and $\Lambda$, so that - if $\mathcal{V}$ is inner stabilisable $-F_{\Omega}$ stabilises the pair $\left(M_{1}^{(1,1)}, M_{2}^{(1,1)}\right)$ and - if $\mathcal{V}$ is outer stabilisable $-\Lambda$ stabilises $\left(M_{1}^{(2,2)}, M_{2}^{(2,2)}\right)$. By using the stability criterion (3) established in [8], two procedures are derived in [15] for the inner and outer stabilisation of output-nulling subspaces. In particular, it is shown that

- the inner stabilisation of the controlled invariant subspace $\mathcal{V}$ requires the solution of a simple LMI;

- the outer stabilisation requires the solution of a bilinear matrix inequality. For its solution, different techniques may be employed. For example, in [15] the so-called sequential linear programming matrix method (SLPMM) developed in [11] is exploited for this purpose, and a simple algorithm is proposed.

We end this section by recalling that, as in the 1-D case, the set of output-nulling controlled invariant subspaces of (10-11) is closed under subspace addition, and the largest output-nulling subspace is denoted by $\mathcal{V}^{\star}$. This subspace can be computed in finite terms as shown in the following algorithm, see [5, Proposition 2.7] and [13, Theorem 2].

Algorithm 3.1. The subspace $\mathcal{V}^{\star}$ can be computed as the $(n-1)$-th term of the monotonic sequence

$$
\left\{\begin{array}{l}
\mathcal{V}_{0}=\mathbb{R}^{n} \\
\mathcal{V}_{i}=\left[\begin{array}{c}
A_{1} \\
A_{2} \\
C
\end{array}\right]^{-1}\left(\left(\mathcal{V}_{i-1}^{2} \times \mathbf{0}_{p}\right)+\operatorname{im}\left[\begin{array}{c}
B_{1} \\
B_{2} \\
D
\end{array}\right]\right) .
\end{array}\right.
$$

\section{SOLUTION OF PROBLEM 2.1}

In the 1-D case, it is an established fact that the solution of the decoupling problem with preview can be expressed in terms of output-nulling and input-containing subspaces of the original system, $[18,4,1]$. In the 2 -D case, this does not seem to be the case, due to the fact that inputcontaining subspaces for 2-D systems as defined in [9] or [14] do not enjoy the useful reachability properties of their one-dimensional counterpart, described for example in $[17$, Chapter 8]. We now analyse the possibility of solving Problem 2.1 by turning it into a decoupling problem of measurable input signals. In fact, the input $w_{i, j}$ in (1) can be thought of as being generated by a 2-D system $\Delta$, whose input is $\hat{w}_{i, j} \triangleq w_{i+N, j+M}$ and whose output is $w_{i, j}$. See in particular Figure 1, where the system governed by (1) is denoted by $\Sigma$, the system $\Delta$ is simply a shift by $N$ and $M$ of the signal indexes $i$ and $j$, respectively, and $\widehat{\Sigma}$ denotes

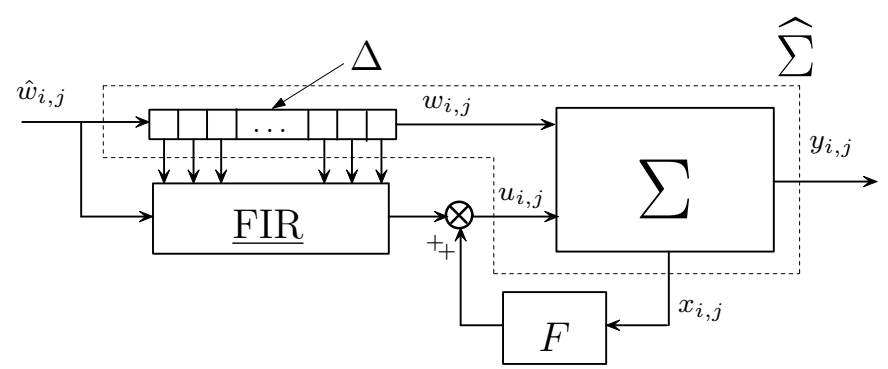

Fig. 1. Block diagram of the compensation scheme.

the extended system obtained by the series connection of $\Sigma$ and $\Delta$. Suppose that a FM realisation is available for the $(N, M)$-shift system $\Delta$, described by 


$$
\begin{aligned}
\xi_{i+1, j+1} & =A_{1}^{\Delta} \xi_{i+1, j}+A_{2}^{\Delta} \xi_{i, j+1}+B_{1}^{\Delta} \hat{w}_{i+1, j}+B_{2}^{\Delta} \hat{w}_{i, j+1}, \\
y_{i, j}^{\Delta} & =C^{\Delta} \xi_{i, j}=\hat{w}_{i-N, j-M}=w_{i, j},
\end{aligned}
$$

where $\xi$ denotes the local state of $\Delta$. The disturbance decoupling problem with preaction can be turned into a measurable signal decoupling problem, where now the plant is given by the series connection $\widehat{\Sigma}$, with input $\left[\begin{array}{c}u \\ \hat{w}\end{array}\right]$, output $y$ and local state $z_{i, j} \triangleq\left[\begin{array}{l}x \\ \xi\end{array}\right]$. The corresponding system matrices are

$$
\begin{aligned}
\widehat{A}_{k} & =\left[\begin{array}{cc}
A_{k} & H_{k} C^{\Delta} \\
0 & A_{k}^{\Delta}
\end{array}\right], \quad \widehat{B}_{k}=\left[\begin{array}{c}
B_{k} \\
0
\end{array}\right], \quad \widehat{H}_{k}=\left[\begin{array}{c}
0 \\
B_{k}^{\Delta}
\end{array}\right] \quad k=1,2, \\
\widehat{C} & =\left[\begin{array}{cc}
C & G C^{\Delta}
\end{array}\right], \quad \widehat{D}=D, \quad \widehat{G}=0 .
\end{aligned}
$$

Problem 2.1 can be recast as a decoupling problem of the measurable signal $\hat{w}_{i, j}$. In fact, suppose we are able to decouple the signal $\hat{w}_{i, j}$ from the output $y_{i, j}$ by means of the control action

$$
u_{i, j}=\widehat{F}\left[\begin{array}{c}
x_{i, j} \\
\xi_{i, j}
\end{array}\right]+S \hat{w}_{i, j}
$$

By partitioning $\widehat{F}=\left[\begin{array}{ll}F_{x} & F_{\xi}\end{array}\right]$, conformably with $\left[\begin{array}{l}x \\ \xi\end{array}\right]$, then the feedback matrix $F$ of the original system can be taken to be equal to $F_{x}$. To find the matrices $S_{k, l}$ in (5), we can compare the solution of the measurable signal decoupling problem (19) with the input structure (5) imposed for our problem. In other words, the matrices $S_{k, l}$ can be derived by matching (5) with (19). For this to be possible, a particular Fornasini-Marchesini realisation is required for the system $\Delta$. Another problem is how to accomodate the stability requirement. Note in particular that this corresponds to a requirement that $F_{x}$ stabilises $\Sigma$, which is quite different to requiring that $\widehat{F}=\left[\begin{array}{ll}F_{x} & F_{\xi}\end{array}\right]$ stabilises $\widehat{\Sigma}$. For the moment, we concentrate on achieving decoupling and constructing an appropriate realisation for $\Delta$. The issue of stability will be considered later.

The 2-D decoupling problem with full information, as solved in $[5,15]$, can be stated for system $\widehat{\Sigma}$ as follows. Find $\widehat{F}$ and $S$ such that $u_{i, j}=\widehat{F} z_{i, j}+S \hat{w}_{i, j}$ decouples the disturbance $\hat{w}$ from the output $y$. This problem is solvable if the inclusion

$$
\operatorname{im}\left[\begin{array}{c}
\widehat{H}_{1} \\
\widehat{H}_{2} \\
\widehat{G}
\end{array}\right] \subseteq\left(\widehat{\mathcal{V}}^{\star} \times \widehat{\mathcal{V}}^{\star} \times \mathbf{0}_{p}\right)+\operatorname{im}\left[\begin{array}{c}
\widehat{B}_{1} \\
\widehat{B}_{2} \\
\widehat{D}
\end{array}\right]
$$

is satisfied, where $\widehat{\mathcal{V}}^{\star}$ is the largest output-nulling of the system $\left(\widehat{A}_{1}, \widehat{A}_{2}, \widehat{B}_{1}, \widehat{B}_{2}, \widehat{C}, \widehat{D}\right) .{ }^{2}$ This solvability condition is contructive. In fact, if (20) is satisfied, there exist matrices $\Phi_{1}, \Phi_{2}$ and $\Psi$ such that [15]

$$
\left[\begin{array}{c}
\widehat{H}_{1} \\
\widehat{H}_{2} \\
\widehat{G}
\end{array}\right]=\left[\begin{array}{ll}
\widehat{V} & 0 \\
0 & \widehat{V} \\
0 & 0
\end{array}\right]\left[\begin{array}{l}
\Phi_{1} \\
\Phi_{2}
\end{array}\right]+\left[\begin{array}{c}
\widehat{B}_{1} \\
\widehat{B}_{2} \\
\widehat{D}
\end{array}\right] \Psi,
$$

where $\widehat{V}$ is a basis matrix for $\widehat{\mathcal{V}}^{\star}$. The solutions $\Phi_{1}, \Phi_{2}$ and $\Psi$ of the linear equation (21) are parameterised in the

\footnotetext{
2 Recall that, if the matrix $\widehat{F}$ is sought within the class of feedback matrices for which the closed-loop pair $\left(\widehat{A}_{1}+\widehat{B}_{1} \widehat{F}, \widehat{A}_{2}+\widehat{B}_{2} \widehat{F}\right)$ is asymptotically stable, a set of solvability conditions is given by (20) and the further condition that $\widehat{\mathcal{V}}^{\star}$ be inner and outer stabilisable.
}

null-space of $\left[\begin{array}{ccc}\widehat{V} & 0 & \widehat{B}_{1} \\ 0 & \widehat{V} & \widehat{B}_{2} \\ 0 & 0 & \widehat{D}\end{array}\right]$. If we take any friend $\widehat{F}$ of $\widehat{\mathcal{V}}^{\star}$, the input $u_{i, j}=\widehat{F} z_{i, j}+S \hat{w}_{i, j}$ achieves exact decoupling without stability. Indeed, by substituting this control input in (1) we obtain

$$
\begin{aligned}
z_{i+1, j+1}= & \left(\widehat{A}_{1}+\widehat{B}_{1} \widehat{F}\right) z_{i+1, j}+\left(\widehat{A}_{2}+\widehat{B}_{2} \widehat{F}\right) z_{i, j+1} \\
& +\widehat{V} \Phi_{1} \hat{w}_{i+1, j}+\widehat{V} \Phi_{2} \hat{w}_{i, j+1} \\
y_{i, j}= & (\widehat{C}+\widehat{D} \widehat{F}) z_{i, j}
\end{aligned}
$$

which is clearly disturbance decoupled, since, given any $\widehat{\mathcal{V}}^{\star}$-valued boundary condition over the separation set $\mathbb{S}_{0}$, we get $z_{i, j} \in \widehat{\mathcal{V}}^{\star}$ and $y_{i, j}=0$ for all $i, j$ such that $i+j \geq 0$.

As aforementioned, in order to find the matrices $S_{k, l}$, the expressions (5) and (19) must be matched. To this end, it suffices to ensure that the local state $\xi_{i, j}$ of $\Delta$ incorporates the values of the disturbance $w$ for indexes in the rectangle $\mathbb{B}_{i, j} \triangleq\{(k, l) \in \mathbb{Z} \times \mathbb{Z} \mid i \leq k \leq N, j \leq l \leq M\}$, excluding $w_{i+N, j+M}$, which can be directly used as an input of the compensator. This is achieved by finding a realisation for $\Delta$ of order $q \triangleq d[(N+1)(M+1)-1]$, where we recall that $d$ is the dimension of the disturbance, so that its local state is given by the values of $w$ on $\mathbb{B}_{i, j} \backslash\{(i+N, j+M)\}$. A realisation meeting this requirement is given as follows. For $P \in \mathbb{N}$, define

$N_{P}^{\prime}=\left[\begin{array}{ccccc}0 & 0 & \ldots & 0 & 0 \\ I_{d} & 0 & \ldots & 0 & 0 \\ 0 & I_{d} & \ldots & 0 & 0 \\ \vdots & \vdots & \ddots & \vdots & \vdots \\ 0 & 0 & \ldots & I_{d} & 0\end{array}\right], N_{P}^{\prime \prime}=\left[\begin{array}{ccccc}I_{d} & 0 & \ldots & 0 & 0 \\ 0 & 0 & \ldots & 0 & 0 \\ 0 & 0 & \ldots & 0 & 0 \\ \vdots & \vdots & \ddots & \vdots & \vdots \\ 0 & 0 & \ldots & 0 & 0\end{array}\right], V_{P}=\left[\begin{array}{c}I_{d} \\ 0 \\ 0 \\ \vdots \\ 0\end{array}\right]$,

where $N_{P}^{\prime}, N_{P}^{\prime \prime} \in \mathbb{R}^{P \cdot d \times P \cdot d}$ and $V_{P} \in \mathbb{R}^{P \cdot d \times d}$. The matrices

$$
\begin{aligned}
A_{1}^{\Delta} & =\left[\begin{array}{ccccc}
N_{M}^{\prime} & 0 & 0 & \ldots & 0 \\
0 & N_{M+1}^{\prime} & 0 & \ldots & 0 \\
0 & 0 & N_{M+1}^{\prime} & \ldots & 0 \\
\vdots & \vdots & \vdots & \ddots & \vdots \\
0 & 0 & 0 & \ldots & N_{M+1}^{\prime}
\end{array}\right], \quad B_{1}^{\Delta}=\left[\begin{array}{c}
V_{M} \\
0 \\
0 \\
\vdots \\
0
\end{array}\right], \\
A_{2}^{\Delta} & =\left[\begin{array}{cccccc}
0 & 0 & 0 & \ldots & 0 & 0 \\
0 & 0 & 0 & \ldots & 0 & 0 \\
0 & N_{M+1}^{\prime \prime} & 0 & \ldots & 0 & 0 \\
0 & 0 & N_{M+1}^{\prime \prime} & \ldots & 0 & 0 \\
\vdots & \vdots & \vdots & \ddots & \vdots & \vdots \\
0 & 0 & 0 & \ldots & N_{M+1}^{\prime \prime}
\end{array}\right], \quad B_{2}^{\Delta}=\left[\begin{array}{c}
0 \\
V_{M+1} \\
0 \\
0 \\
\vdots \\
0
\end{array}\right], \\
C^{\Delta} & =\left[\begin{array}{lllll}
0 & 0 & \ldots & 0 & I_{d}
\end{array}\right]
\end{aligned}
$$

are a realisation of $\Delta$. In fact, a direct check shows that 


$$
\begin{aligned}
\xi_{i, j}^{1} & =\hat{w}_{i, j-1} \\
\xi_{i, j}^{2} & =\hat{w}_{i, j-2} \\
& \vdots \\
\xi_{i, j}^{M} & =\hat{w}_{i, j-M} \\
\hline \xi_{i, j}^{M+1} & =\hat{w}_{i-1, j} \\
\xi_{i, j}^{M+2} & =\hat{w}_{i-1, j-1} \\
& \vdots \\
\xi_{i, j}^{2 M+2} & =\hat{w}_{i-1, j-M} \\
\hline \xi_{i, j}^{2 M+3} & =\hat{w}_{i-2, j} \\
\xi_{i, j}^{2 M+4} & =\hat{w}_{i-2, j-1} \\
& \vdots \\
\xi_{i, j}^{3 M+4} & =\hat{w}_{i-2, j-M} \\
\xi_{i, j}^{3 M+5} & =\hat{w}_{i-3, j} \\
& \vdots \\
\xi_{i, j}^{N M+N+M} & =\hat{w}_{i-N, j-M} \\
y_{i, j}^{\Delta} & =x_{i, j}^{N M+N+M}=\hat{w}_{i-N, j-M}=w_{i, j} .
\end{aligned}
$$

If the decoupling problem of the measurable signal $\hat{w}_{i, j}$ is solvable for $\widehat{\Sigma}$, a control function having the structure (19) can be found to achieve perfect decoupling. By partitioning $F_{\xi}=\left[\begin{array}{lllll}F_{\xi}^{1} & F_{\xi}^{2} & F_{\xi}^{3} \ldots F_{\xi}^{N M+N+M}\end{array}\right]$ conformably with $\xi=$ $\left[\begin{array}{c}\xi^{1} \\ \vdots \\ \xi^{N M+N+M}\end{array}\right]$, by comparing (5) with (19), it follows that $F=F_{x}, S_{N, M}=S, S_{N-1, M}=F_{\xi}^{M+1}, S_{N, M-1}=F_{\xi}^{1}$, $S_{N-1, M-1}=F_{\xi}^{M+2}, S_{N-2, M-1}=F_{\xi}^{2 M+3}, \ldots$, solve Problem 2.1.

Now we turn our attention to the stability requirement. Requiring that $\widehat{\mathcal{V}}^{\star}$ is inner and outer stabilisable, as one might expect at first sight, due to the analogy with the measurable signal decoupling problem, is not correct in this case, since $F_{\xi}$ cannot be used to stabilise $\Sigma$. Here we want to show that the stability condition required for the solution of Problem 2.1 can be stated in terms of the stabilisability of the largest output-nulling subspace $\mathcal{V}^{\star}$ of the system $\left(A_{1}, A_{2}, B_{1}, B_{2}, C, D\right)$. To this end, we first present the following lemma, where the relation between $\mathcal{V}^{\star}$ and $\widehat{\mathcal{V}}^{\star}$ is established.

Lemma 4.1. The following identity holds:

$$
\widehat{\mathcal{V}}^{\star} \cap \operatorname{im}\left[\begin{array}{c}
I_{n} \\
0_{q \times n}
\end{array}\right]=\mathcal{V}^{\star} \times \mathbf{0}_{q}
$$

Proof: First, we show that the subspace on the left-hand side of (22) contains that on the right-hand side, i.e., $\widehat{\mathcal{V}}^{\star} \supseteq$ $\mathcal{V}^{\star} \times \mathbf{0}_{q}$. Consider the two sequences of subspaces $\left\{\mathcal{V}_{i}\right\}_{i \in \mathbb{N}}$ and $\left\{\widehat{\mathcal{V}}_{i}\right\}_{i \in \mathbb{N}}$ in Algorithm 3.1 converging respectively to $\mathcal{V}^{\star}$ and to $\widehat{\mathcal{V}}^{\star}$. By induction, suppose that $\widehat{\mathcal{V}}_{i-1} \supseteq \mathcal{V}_{i-1} \times \mathbf{0}_{q}$. Take $\left[\begin{array}{l}x \\ 0\end{array}\right] \in \mathcal{V}_{i} \times \mathbf{0}_{q}$. Since $x \in \mathcal{V}_{i}$ we find that there exist $\xi_{1}, \xi_{2} \in \mathcal{V}_{i-1}$ and $\omega \in \mathbb{R}^{m}$ such that

$$
\left[\begin{array}{c}
A_{1} \\
A_{2} \\
C
\end{array}\right] x=\left[\begin{array}{c}
\xi_{1} \\
\xi_{2} \\
0
\end{array}\right]+\left[\begin{array}{c}
B_{1} \\
B_{2} \\
D
\end{array}\right] \omega
$$

Now, we show that $\left[\begin{array}{l}x \\ 0\end{array}\right] \in \widehat{\mathcal{V}}_{i}$. In fact

$$
\begin{aligned}
{\left[\begin{array}{cc}
A_{1} & H_{1} C^{\Delta} \\
0 & A_{1}^{\Delta} \\
\hline A_{2} & H_{2} C^{\Delta} \\
0 & A_{2}^{\Delta} \\
\hline C & G C^{\Delta}
\end{array}\right]\left[\begin{array}{l}
x \\
0
\end{array}\right] } & =\left[\begin{array}{c}
A_{1} x \\
0 \\
\frac{0}{A_{2} x} \\
0 \\
\hline C x
\end{array}\right]=\left[\begin{array}{c}
\xi_{1}+B_{1} \omega \\
\frac{0}{\xi_{2}+B_{2} \omega} \\
\frac{0}{D u}
\end{array}\right] \\
& \in\left(\mathcal{V}_{i-1} \times \mathbf{0}_{q}\right)^{2} \times \mathbf{0}_{p}+\operatorname{im}\left[\begin{array}{c}
\widehat{B}_{1} \\
\widehat{B}_{2} \\
\widehat{D}
\end{array}\right] .
\end{aligned}
$$

From the inductive assumption $\mathcal{V}_{i-1} \times \mathbf{0}_{q} \subseteq \widehat{\mathcal{V}}_{i-1}$ it follows that $\left[\begin{array}{c}\widehat{A}_{1} \\ \widehat{A}_{2} \\ \widehat{C}\end{array}\right]\left[\begin{array}{l}x \\ 0\end{array}\right] \subseteq\left(\widehat{\mathcal{V}}_{i-1}^{2} \times \mathbf{0}_{p}\right)+\operatorname{im}\left[\begin{array}{c}\widehat{B}_{1} \\ \widehat{B}_{2} \\ \widehat{D}\end{array}\right]$, and hence $\left[\begin{array}{l}x \\ 0\end{array}\right] \in \widehat{\mathcal{V}}_{i}$. Now, we prove the opposite inclusion. First, let

$$
\widehat{\mathcal{V}}_{i-1} \cap \operatorname{im}\left[\begin{array}{c}
I_{n} \\
0_{q \times n}
\end{array}\right] \subseteq \mathcal{V}_{i-1} \times \mathbf{0}_{q}
$$

To prove that the same is true for $i$, let $\left[\begin{array}{l}x \\ 0\end{array}\right] \in \widehat{\mathcal{V}}_{i} \cap$ $\operatorname{im}\left[\begin{array}{c}I_{n} \\ 0_{q \times n}\end{array}\right]$, so that by Algorithm 3.1

$$
\left[\begin{array}{cc}
A_{1} & H_{1} C^{\Delta} \\
0 & A_{1}^{\Delta} \\
\hline A_{2} & H_{2} C^{\Delta} \\
0 & A_{2}^{\Delta} \\
\hline C & G C^{\Delta}
\end{array}\right]\left[\begin{array}{l}
x \\
0
\end{array}\right] \in\left(\widehat{\mathcal{V}}_{i-1}^{2} \times \mathbf{0}_{p}\right)+\operatorname{im}\left[\begin{array}{c}
B_{1} \\
0 \\
\hline B_{2} \\
0 \\
\hline D
\end{array}\right] .
$$

On the other hand, by (23) we have

$$
\left(\widehat{\mathcal{V}}_{i-1} \cap \operatorname{im}\left[\begin{array}{c}
I_{n} \\
0_{q \times n}
\end{array}\right]\right)^{2}+\operatorname{im}\left[\begin{array}{c}
\widehat{B}_{1} \\
\widehat{B}_{2} \\
\widehat{D}
\end{array}\right] \subseteq\left(\mathcal{V}_{i-1} \times \mathbf{0}_{p}\right)^{2}+\operatorname{im}\left[\begin{array}{c}
\widehat{B}_{1} \\
\widehat{B}_{2} \\
\widehat{D}
\end{array}\right]
$$

which leads to

$$
\left[\begin{array}{cc}
A_{1} & H_{1} C^{\Delta} \\
0 & A_{1}^{\Delta} \\
\hline A_{2} & H_{2} C^{\Delta} \\
0 & A_{2}^{\Delta} \\
\hline C & G C^{\Delta}
\end{array}\right]\left[\begin{array}{l}
x \\
0
\end{array}\right]=\left[\begin{array}{c}
A_{1} x \\
0 \\
\hline A_{2} x \\
0 \\
\hline C x
\end{array}\right] \in\left(\mathcal{V}_{i-1} \times \mathbf{0}_{q}\right)^{2} \times \mathbf{0}_{p}+\mathrm{im}\left[\begin{array}{c}
B_{1} \\
0 \\
B_{2} \\
0 \\
\hline D
\end{array}\right] .
$$

This in turn implies $\left[\begin{array}{c}A_{1} \\ A_{2} \\ C\end{array}\right] x \in\left(\mathcal{V}_{i-1}^{2} \times \mathbf{0}_{p}\right)+\operatorname{im}\left[\begin{array}{c}B_{1} \\ B_{2} \\ D\end{array}\right]$ and so, by Algorithm 3.1, it follows that $x \in \mathcal{V}_{i}$, so that $\left[\begin{array}{l}x \\ 0\end{array}\right] \in \mathcal{V}_{i} \times \mathbf{0}_{q}$.

Armed with Lemma 4.1, we can now provide a complete solution to Problem 2.1.

Theorem 4.1. Problem 2.1 is solvable if

(i) $\operatorname{im}\left[\begin{array}{l}\widehat{H}_{1} \\ \widehat{H}_{2}\end{array}\right] \subseteq\left(\widehat{\mathcal{V}}^{\star} \times \widehat{\mathcal{V}}^{\star}\right)+\left[\begin{array}{l}\widehat{B}_{1} \\ \widehat{B}_{2}\end{array}\right]$ ker $D$;

(ii) $\mathcal{V}^{\star}$ is inner and outer stabilisable. 
Proof: First, observe that the structural condition (i) is just a simplified way of writing (20), due to the fact that $\widehat{G}$ is zero. Now we show (ii). By virtue of Lemma 4.1, it follows that $\widehat{\mathcal{V}}^{\star}$ can be written as

$$
\widehat{\mathcal{V}}^{\star}=\operatorname{im}\left[\begin{array}{ll}
V & V_{2} \\
0 & V_{3}
\end{array}\right],
$$

where $V$ is a basis matrix of $\mathcal{V}^{\star}$ and $V_{3}$ is of full columnrank. Since $\widehat{\mathcal{V}}^{\star}$ is output-nulling for $\widehat{\Sigma}$, any output-nulling friend $\widehat{F}=\left[\begin{array}{ll}F_{x} & F_{\xi}\end{array}\right]$ of $\widehat{\mathcal{V}}^{\star}$ is such that the matrix associated with the internal dynamics on $\widehat{\mathcal{V}}^{\star}$ satisfies

$$
\begin{aligned}
& {\left[\begin{array}{cc}
A_{1}+B_{1} F_{x} & H_{1} C^{\Delta}+B_{1} F_{\xi} \\
0 & A_{1}^{\Delta} \\
\hline A_{2}+B_{2} F_{x} & H_{2} C^{\Delta}+B_{2} F_{\xi} \\
0 & A_{2}^{\Delta} \\
\hline C+D F_{x} & G C^{\Delta}+D F_{\xi}
\end{array}\right]\left[\begin{array}{ll}
V & V_{2} \\
0 & V_{3}
\end{array}\right]} \\
& =\left[\begin{array}{cc|cc}
V & V_{2} & 0 & 0 \\
0 & V_{3} & 0 & 0 \\
\hline 0 & 0 & V & V_{2} \\
0 & 0 & 0 & V_{3} \\
\hline 0 & 0 & 0 & 0
\end{array}\right]\left[\begin{array}{ll}
X_{11} & X_{12} \\
X_{21} & X_{22} \\
\hline X_{31} & X_{32} \\
X_{41} & X_{42}
\end{array}\right] .
\end{aligned}
$$

From (25) we find the two identities $V_{3} X_{21}=0$ and $V_{3} X_{41}=0$, which lead to $X_{21}=0$ and to $X_{41}=0$ since $V_{3}$ is full column-rank. From the identities $A_{1}^{\Delta} V_{3}=V_{3} X_{22}$ and $A_{2}^{\Delta} V_{3}=V_{3} X_{42}$, which follow from (25), we find that $\operatorname{im} V_{3}$ is an $\left(A_{1}^{\Delta}, A_{2}^{\Delta}\right)$-invariant subspace. Let us now write (13) for the output-nulling $\widehat{\mathcal{V}}^{\star}$ in the partitioned form

$$
\begin{aligned}
& {\left[\begin{array}{cc}
A_{1} & H_{1} C^{\Delta} \\
0 & A_{1}^{\Delta} \\
\hline A_{2} & H_{2} C^{\Delta} \\
0 & A_{2}^{\Delta} \\
\hline C & G C^{\Delta}
\end{array}\right]\left[\begin{array}{ll}
V & V_{2} \\
0 & V_{3}
\end{array}\right]=} \\
& {\left[\begin{array}{cc|cc}
V & V_{2} & 0 & 0 \\
0 & V_{3} & 0 & 0 \\
\hline 0 & 0 & V & V_{2} \\
0 & 0 & 0 & V_{3} \\
\hline 0 & 0 & 0 & 0
\end{array}\right]\left[\begin{array}{cc}
X_{11} & X_{12} \\
0 & X_{22} \\
X_{31} & X_{32} \\
0 & X_{42}
\end{array}\right]+\left[\begin{array}{c}
B_{1} \\
0 \\
\hline B_{2} \\
0 \\
D
\end{array}\right]\left[\begin{array}{ll}
\Omega_{1} & \Omega_{2}
\end{array}\right] .}
\end{aligned}
$$

Since $\mathcal{V}^{\star}$ is inner and outer stabilisable, we can find an output-nulling friend $F_{x}$ of $\mathcal{V}^{\star}$ such that

$$
\begin{aligned}
\left(A_{1}+B_{1} F_{x}\right) V & =V \bar{X}_{1} \\
\left(A_{2}+B_{2} F_{x}\right) V & =V \bar{X}_{2} \\
\left(C+D F_{x}\right) V & =0
\end{aligned}
$$

for some $\bar{X}_{1}$ and $\bar{X}_{2}$, and the pair $\left(A_{1}+B_{1} F_{x}, A_{2}+\right.$ $\left.B_{2} F_{x}\right)$ is asymptotically stable; i.e., $F_{x}$ internally and externally stabilises $\mathcal{V}^{\star}$. It follows, in particular, that the pair $\left(\bar{X}_{1}, \bar{X}_{2}\right)$ is asymptotically stable. Such a matrix $F_{x}$ is associated with another matrix $\Omega$ for which

$$
\left[\begin{array}{c}
A_{1} \\
A_{2} \\
C
\end{array}\right] V=\left[\begin{array}{ll}
V & 0 \\
0 & V \\
0 & 0
\end{array}\right]\left[\begin{array}{c}
\bar{X}_{1} \\
\bar{X}_{2}
\end{array}\right]+\left[\begin{array}{c}
B_{1} \\
B_{2} \\
D
\end{array}\right] \Omega
$$

with $\Omega=-F V$. Take $\Omega_{1}=\Omega, X_{11}=\bar{X}_{1}$ and $X_{31}=\bar{X}_{2}$ in (25), and compute $X_{12}, X_{22}, X_{32}, X_{42}, \Omega_{2}$ by

$$
\left[\begin{array}{c}
X_{12} \\
X_{22} \\
\hline X_{32} \\
X_{42} \\
\hline \Omega_{2}
\end{array}\right]=\left[\begin{array}{cc|cc|c}
V & V_{2} & 0 & 0 & B_{1} \\
0 & V_{3} & 0 & 0 & 0 \\
\hline 0 & 0 & V & V_{2} & B_{2} \\
0 & 0 & 0 & V_{3} & 0 \\
\hline 0 & 0 & 0 & 0 & D
\end{array}\right]^{\dagger}\left[\begin{array}{c}
A_{1} V_{2}+H_{1} C^{\Delta} V_{3} \\
\frac{A_{1}^{\Delta} V_{3}}{A_{2} V_{2}+H_{2} C^{\Delta} V_{3}} \\
\frac{A_{2}^{\Delta} V_{3}}{C V_{2}+G C^{\Delta} V_{3}}
\end{array}\right]
$$

Now, the friend $\widehat{F}=\left[\begin{array}{ll}F_{x} & F_{\xi}\end{array}\right]$ of $\widehat{\mathcal{V}}^{\star}$ can be computed as a solution of the equation

$\left[\begin{array}{ll}\Omega_{1} & \Omega_{2}\end{array}\right]=-\left[\begin{array}{ll}F_{x} & F_{\xi}\end{array}\right]\left[\begin{array}{ll}V & V_{2} \\ 0 & V_{3}\end{array}\right]=-\left[\begin{array}{ll}F_{x} V & F_{x} V_{2}+F_{\xi} V_{3}\end{array}\right]$.

The first component $F_{x}$ of $\widehat{F}$ satisfies $\Omega_{1}=-F_{x} V$, so that it stabilises $\mathcal{V}^{\star}$ internally and externally. The second component $F_{\xi}$ can be computed as

$$
F_{\xi}=-\left(\Omega_{2}+F_{x} V_{2}\right)\left(V_{3}^{\top} V_{3}\right)^{-1} V_{3}^{\top}
$$

Notice that in Theorem 4.1, the structural condition is given in terms of the output-nulling $\widehat{\mathcal{V}}^{\star}$, while the stability condition is expressed in terms of the inner and outer stabilisability of $\mathcal{V}^{\star}$.

Example 4.1. Let (10-11) be defined over $\mathbb{N} \times \mathbb{N}$ with

$$
\begin{aligned}
& A_{1}=\left[\begin{array}{ccccc}
-0.03 & 0 & 0.04 & 0 & 0 \\
0 & -0.02 & 0.1 & 0 & 0 \\
0 & 0 & -0.07 & 0 & 0 \\
0 & 0 & -0.02 & 0.06 & 0.05 \\
-0.1 & -0.09 & 0.08 & 0.03 & 0.03
\end{array}\right], B_{1}=\left[\begin{array}{cc}
0 & -9 \\
0 & 5 \\
0 & 0 \\
0 & 0 \\
0 & -5
\end{array}\right], H_{1}=\left[\begin{array}{c}
-5 \\
8 \\
0 \\
-2 \\
-4
\end{array}\right] \text {, } \\
& A_{2}=\left[\begin{array}{ccccc}
-0.12 & 0 & 0 & 0 & 0 \\
0 & 0 & 0.04 & 0.08 & 0.1 \\
0 & 0 & 0.04 & 0.18 & 0.08 \\
0 & 0 & 0 & 0 & 0 \\
0 & 0 & 0 & -0.06 & -0.16
\end{array}\right], B_{2}=\left[\begin{array}{cc}
0 & 0 \\
0 & -9 \\
1 & -8 \\
0 & 0 \\
0 & 5
\end{array}\right], H_{2}=\left[\begin{array}{c}
0 \\
-9 \\
5 \\
0 \\
0
\end{array}\right] \text {, }
\end{aligned}
$$$$
C=\left[\begin{array}{lllll}
0 & 0 & 0 & -3 & 0
\end{array}\right], \quad D=\left[\begin{array}{ll}
0 & 0
\end{array}\right], \quad G=0 .
$$

The conditions associated with this system are random assignments of the local state over the region $(\mathbb{N} \times\{0\}) \cup$ $(\{0\} \times \mathbb{N})$. By using Algorithm 3.1 for the computation of the largest output-nulling subspace $\mathcal{V}^{\star}$ of the system $\left(A_{1}, A_{2}, B_{1}, B_{2}, C, D\right)$ we get

$$
\mathcal{V}^{\star}=\operatorname{im} V, \quad \text { where } \quad V=\left[\begin{array}{ccc}
-1 & 0 & 0 \\
0 & -1 & 0 \\
0 & 0 & 5 \\
0 & 0 & 0 \\
0 & 0 & 2
\end{array}\right] \text {. }
$$

A simple check shows that the structural condition $\operatorname{im}\left[\begin{array}{c}H_{1} \\ H_{2} \\ G\end{array}\right] \subseteq\left(\mathcal{V}^{\star} \times \mathcal{V}^{\star} \times \mathbf{0}_{p}\right)+\operatorname{im}\left[\begin{array}{c}B_{1} \\ B_{2} \\ D\end{array}\right]$ for the solution of the measurable signal decoupling problem is not satisfied in this case. As such, the decoupling problem cannot be solved using the techniques described herein for the control structure $u_{i, j}=F x_{i, j}+S w_{i, j}$, nor any other existing geometric technique for $2-\mathrm{D}$ systems. Suppose now that the control law is allowed to be in the form (5) with $N=2$ and $M=1$, so that $u_{i, j}=F x_{i, j}+S_{0,0} w_{i, j}+S_{0,1} w_{i, j+1}+$ $S_{1,0} w_{i+1, j}+S_{1,1} w_{i+1, j+1}+S_{2,0} w_{i+2, j}+S_{2,1} w_{i+2, j+1}$. First, we find a stabilising output-nulling friend $F$ of $\mathcal{V}^{\star}$. In this case the null-space of $W=\left[\begin{array}{ccc}V & 0 & B_{1} \\ 0 & V & B_{2} \\ 0 & 0 & D\end{array}\right]$ is zero. As such, the unique solution $\left(X_{1}, X_{2}\right)$ to $\left[\begin{array}{c}X_{1} \\ X_{2} \\ \Omega\end{array}\right]=W^{\dagger}\left[\begin{array}{c}A_{1} \\ A_{2} \\ C\end{array}\right] V$ must satisfy condition (3). Indeed, the solution 


$$
\begin{aligned}
X_{1} & =\left[\begin{array}{ccc}
0.150 & 0.162 & 0.880 \\
-0.100 & -0.110 & -1.100 \\
0 & 0 & -0.070
\end{array}\right], \quad X_{2}\left[\begin{array}{ccc}
-0.120 & 0 & 0 \\
0.18 & 0.162 & 0.680 \\
0.05 & 0.0450 & 0.140
\end{array}\right], \\
\Omega & =\left[\begin{array}{ccc}
-0.410 & -3.690 & -1.300 \\
-0.020 & -0.0180 & -0.120
\end{array}\right],
\end{aligned}
$$

is such that the pair $\left(X_{1}, X_{2}\right)$ is asymptotically stable, i.e., it satisfies (3), so that $F$ stabilises $\mathcal{V}^{\star}$ internally. With this choice we find

$$
F=-\Omega\left(V^{\top} V\right)^{-1} V^{\top}=\left[\begin{array}{ccccc}
-0.410 & -0.369 & 0.2241 & 0 & 0.0897 \\
-0.020 & -0.0180 & 0.0207 & 0 & 0.0083
\end{array}\right] .
$$

A direct check shows that the pair $\left(A_{1}+B_{1} F, A_{2}+B_{2} F\right)$ is asymptotically stable as it satisfies $(3)$, so that $F$ stabilises $\mathcal{V}^{\star}$ externally as well. A model for system $\Delta$ is

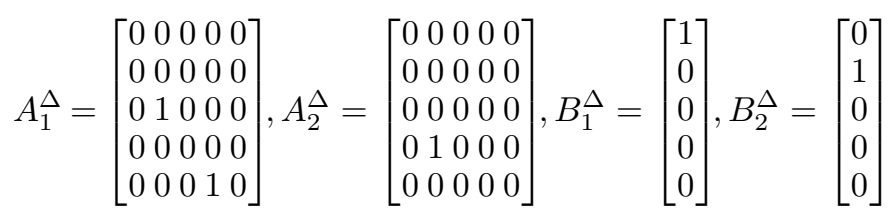
$C^{\Delta}=\left[\begin{array}{lllll}0 & 0 & 0 & 0 & 1\end{array}\right]$.

By applying Algorithm 3.1 to the series $\widehat{\Sigma}$ of $\Sigma$ and $\Delta$ it is easy to see that a basis matrix for the subspace $\widehat{\mathcal{V}}^{\star}$ can be expressed as in (24), where

$V_{2}=\left[\begin{array}{ccccc}0 & 0 & 0 & 0 & 0 \\ 0 & 0 & 0 & 0 & 0 \\ 0 & 0 & 0 & 0 & 0 \\ 0 & 0 & 0 & 0 & 0 \\ 0.9997 & 0 & 0 & 0 & 0\end{array}\right]$ and $V_{3}=\left[\begin{array}{ccccc}0 & 1 & 0 & 0 & 0 \\ 0 & 0 & 1 & 0 & 0 \\ 0 & 0 & 0 & -1 & 0 \\ 0 & 0 & 0 & 0 & -1 \\ 0.0250 & 0 & 0 & 0 & 0\end{array}\right]$.

A direct check shows that conditions ( $i$-ii) in Theorem 4.1 are satisfied, so that an input function in the form (5) with $N=2$ and $M=1$ exists such that the overall system is disturbance decoupled from the input $\hat{w}$ to the output $y$. Our aim now is to find the matrices $S_{k, l},(k, l) \in[0,2] \times$ $[0,1]$ to be employed for the synthesis of the FIR system. Let us exploit (26) for the computation of $X_{12}, X_{22}, X_{32}$, $X_{42}$ and $\Omega_{2}$, so that $(27)$ can be used to compute $F_{\xi}$ :

$$
F_{\xi}=\left[\begin{array}{ccccc}
0 & 0 & 0 & -164 & -39.2662 \\
0 & 0 & 0 & -8 & -0.8910
\end{array}\right]
$$

As a result, the gain matrices of the FIR system are $S_{0,0}=\left[\begin{array}{c}-39.26624 \\ -0.8910\end{array}\right], S_{0,1}=\left[\begin{array}{c}-164 \\ -8\end{array}\right], S_{1,0}=S_{1,1}=S_{2,0}=0$, while matrix $S_{2,1}$ can be computed by solving equation (21) written with respect to $\widehat{\Sigma}$ in $\Psi$ and by taking $S_{2,1}=$ $-\Psi$. In this case, $S_{2,1}=0$. It follows that the input $u_{i, j}=F x_{i, j}+S_{0,0} w_{i, j}+S_{0,1} w_{i, j+1}$ solves the disturbance decoupling problem. Clearly, the same result would have been found by choosing $N=0$ and $M=1$. This example shows that the possibility of enriching the control law (5) with the previewed terms $\varphi_{i, j}$ enlarges the possibilities of decoupling exactly the disturbance input $w$ from the output $y$.

Let $\Sigma$ be subject to the randomly generated input $w$ depicted within the interval $[0,20] \times[0,20]$ in Figure 2 and with randomly generated boundary conditions for $\Sigma$. Asymptotic stability of the closed-loop guarantees that the output approaches zero as the index $(i, j)$ evolves away from the axes, see Figure 3.

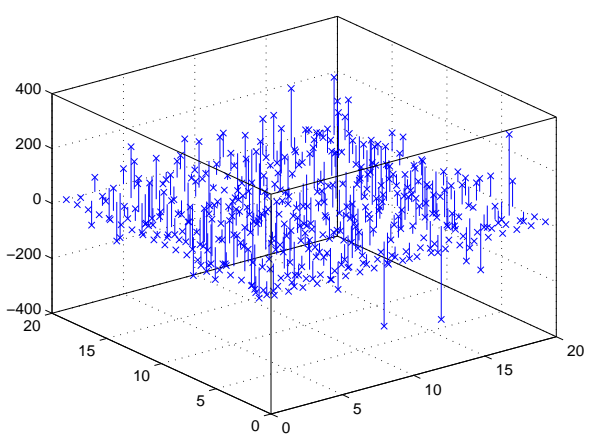

Fig. 2. Disturbance $w$ in the bounded frame $[0,20] \times[0,20]$.

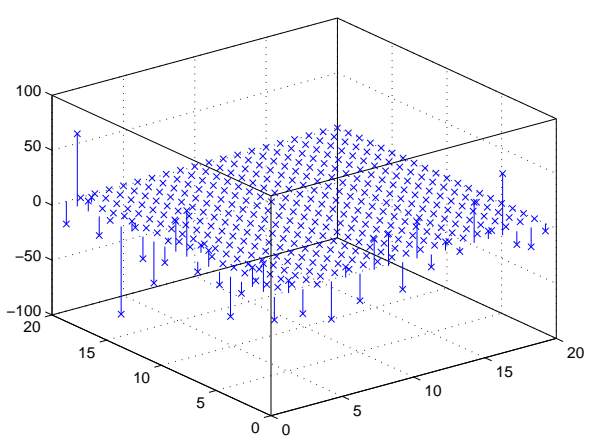

Fig. 3. Output $y_{i, j}$ in the interval $[0,20] \times[0,20]$ for nonzero boundary conditions.

In order to see that as the index $(i, j)$ evolves away from the axis the output $y_{i, j}$ decreases in an exponential fashion, Figure 4 shows the base 10 logarithm of $\left|y_{i, i}\right|$ for $i \in[0,20]$.

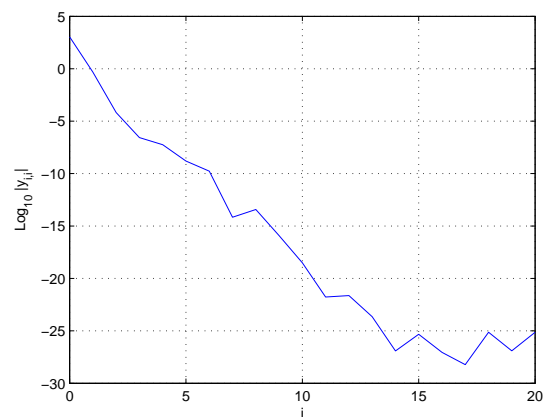

Fig. 4. Logarithm of the output $\left|y_{i, i}\right|$ for $i \in[0,20]$.

If on the other hand we assume zero boundary conditions, the disturbance signal $w$ in Figure 2 leads to the output $y$ depicted in Figure 5, which shows perfect decoupling (to within numerical noise).

\section{REFERENCES}

[1] F. Barbagli, G. Marro, and D. Prattichizzo, "Generalized signal decoupling problem with stability for discrete time systems," J. Opt. Theory Appl., vol. 111, no. 1, October 2001.

[2] G. Basile and G. Marro. Controlled and conditioned invariant subspaces in linear system theory. J. Opt. Theory Appl., 3(5):306-315, 1969. 


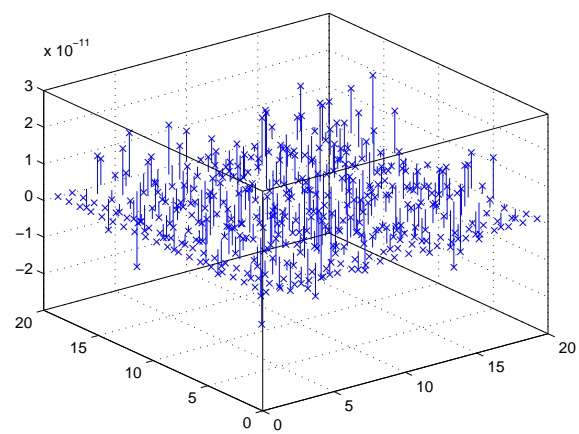

Fig. 5. Output $y_{i, j}$ obtained with boundary conditions at zero. Note that $\left|y_{i, j}\right| \sim 10^{-11}$.

[3] G. Basile and G. Marro, "Self-bounded controlled invariant subspaces: a straightforward approach to constrained controllability," J. Opt. Theory Appl., vol. 38, no. 1, pp. 71-81, 1982.

[4] M. Bonilla Estrada and M. Malabre, "Necessary and sufficient conditions for disturbance decoupling with stability using PID control laws," IEEE Trans. Autom. Contr., vol. 44, no. 6, pp. 1311-1315, June 1999.

[5] G. Conte and A. Perdon. A geometric approach to the theory of 2-D systems. IEEE Trans. Autom. Contr., AC-33(10):946-950, 1988.

[6] E. Fornasini and G. Marchesini. Doubly-Indexed Dynamical Systems: State-Space Models and Structural Properties. Mathematical System Theory, 12:59-72, 1978.

[7] E. Fornasini and G. Marchesini. Stability analysis of 2-D systems. IEEE Transactions on Circuits and Systems, CAS-27(12):1210-1217, 1980.

[8] H. Kar and V. Sigh. Stability of 2-D systems described by the Fornasini-Marchesini first model. IEEE Trans. Sign. Process., 51(6):1675-1676, 2003.

[9] A. Karamanciog̃lu and F.L. Lewis. A geometric approach to 2-D implicit systems. Proc. 29th Conf. Dec. Contr., Honolulu, Hawaii, December 1990.

[10] A. Karamanciog̃lu and F.L. Lewis. Geometric theory for the singular Roesser model. IEEE Trans. Autom. Contr., AC-37(6):801-806, 1992.

[11] F. Leibfritz. An LMI-based algorithm for designing suboptimal static $\mathcal{H}_{2} / \mathcal{H}_{\infty}$ output feedback controllers. SIAM J. Contr. Optim., 39(6):1711-1735, 2001.

[12] M. Malabre, J. Martínez-García, and B. Del-MuroCuéllar, "On the fixed poles for disturbance rejection," Automatica, vol. 33, no. 6, pp. 1209-1211, 1997.

[13] L. Ntogramatzidis, and M. Cantoni. New Results and Applications of the Geometric Approach to TwoDimensional Systems. Proc. 45th Conf. Dec. Contr., San Diego, CA, USA, December 13-15, 2006.

[14] L. Ntogramatzidis, and M. Cantoni. Conditioned Invariance and Unknown-Input Observation for TwoDimensional Fornasini-Marchesini Models. In Proc. Europ. Contr. Conf. 2007, Kos, Greece, July 2-5, 2007.

[15] L. Ntogramatzidis, M. Cantoni and R. Yang. A Geometric Approach with Stability for Two-Dimensional Systems. To appear in $46^{\text {th }}$ Conf. Dec. Contr., New
Orleans, LA, USA, December 12-14, 2007.

[16] R.P. Roesser. A discrete state-space model for linear image processing. IEEE Trans. Autom. Contr., AC20(1):1-10, 1975.

[17] H.L. Trentelman, A.A. Stoorvogel, and M. Hautus. Control theory for linear systems. Communications and Control Engineering. Springer, Great Britain, 2001.

[18] J. Willems, "Feedforward control, PID control laws, and almost invariant subspaces," Syst. Contr. Lett., vol. 1, no. 4, pp. 277-282, 1982.

[19] W.M. Wonham and A.S. Morse. Decoupling and pole assignment in linear multivariable systems: a geometric approach. SIAM J. Contr., 8(1):1-18, 1970. 